\title{
The mechanics of the Tollmien-Schlichting wave
}

\author{
By PETER G. BAINES ${ }^{1}$, SHARAN J. MAJUMDAR ${ }^{2}$ \\ AND HUMIO MITSUDERA ${ }^{1 \dagger}$ \\ ${ }^{1}$ CSIRO Division of Atmospheric Research, Aspendale, Australia \\ ${ }^{2}$ Department of Applied Mathematics and Theoretical Physics, University of Cambridge, \\ Silver Street, Cambridge, CB3 9EW, UK
}

(Received 4 May 1994 and in revised form 27 September 1995)

We describe a mechanistic picture of the essential dynamical processes in the growing Tollmien-Schlichting wave in a Blasius boundary layer and similar flows. This picture depends on the interaction between two component parts of a disturbance (denoted 'partial modes'), each of which is a complete linear solution in some idealization of the system. The first component is an inviscid mode propagating on the vorticity gradient of the velocity profile with the free-slip boundary condition, and the second, damped free viscous modes in infinite uniform shear with the no-slip condition. There are two families of these viscous modes, delineated by whether the phase lines of the vorticity at the wall are oriented with or against the shear, and they are manifested as resonances in a forced system. The interaction occurs because an initial 'inviscid' disturbance forces a viscous response via the no-slip condition at the wall. This viscous response is large near the resonance associated with the most weakly damped viscous mode, and in the unstable parameter range it has suitable phase at the outer part of the boundary layer to increase the amplitude of the inviscid partial mode by advection.

\section{Introduction}

Although the mathematical theory of the stability of sheared viscous flows was developed many years ago, the physical mechanism behind the process of instability has received relatively little attention, and still remains obscure. Why does the addition of viscous forces to an otherwise stable shear flow sometimes render it unstable? This well-known fact is contrary to normal intuition, and is one of the classic paradoxes of fluid mechanics. Prandtl gave an argument in terms of Reynolds stresses. A brief sketch in terms of vorticity was given by Lighthill (1963, pp. 92-93), and a more detailed explanation was attempted by Betchov \& Criminale (1967). More recently, in a stimulating paper Lindzen (1988, and references therein) has proposed an explanation in terms of the Orr mechanism, but no general consensus as to a clear physical picture has yet emerged. Apart from being interesting in itself, a physical understanding of this viscous instability should be useful in comprehending the more complex phenomena that occur in boundary layers when disturbances grow to become nonlinear and threedimensional.

In this paper we describe a physical mechanism for the two-dimensional instability of a Blasius boundary layer, and similar flows, where the resulting growing disturbance is known as a Tollmien-Schlichting wave. This instability is the initial part of the process of transition to turbulence in boundary layers in common situations such as on aeroplane wings. The essential mathematics is over 60 years old (Schlichting 1968). The mechanism of instability is described here in dynamical terms, and is based on the 
interaction between two idealized modes of the system: an 'inviscid' mode, which is neutral when viscosity is zero and a decaying viscous mode (or modes) that exists in uniform shear. The latter is forced by the former via the no-slip boundary condition at the wall. This dynamical picture is different from that proposed by Lindzen (1988), see $\$ 7$, and is an extension to viscous fluids of the ideas presented in Baines \& Mitsudera (1994) for instability of inviscid shear flows. An early version was presented in Baines \& Mitsudera (1992).

One common complication in flows of this sort is the effect of non-zero vorticity gradient $\left(U_{z z}\right)$ at the critical layers on the flow conditions on either side of it. In this paper we have sidestepped this effect by concentrating on flows where $U_{z z}$ is negligibly small at the critical layer, in the interests of simplifying the physical picture.

The properties of these idealized modes are described in $\S \S 3$ and 4 , and the interaction mechanism of instability in $\S \S 5$ and 6. Apart from their significance for instability, the modes in viscous uniform shear are interesting in their own right, as they provide a simple illustration of properties of disturbances in sheared viscous fluids with critical layers, as described in $\S 4$.

\section{Equations}

We consider a viscous fluid that has velocity profile $U(z)$ in the undisturbed state, where $x$ and $z$ are horizontal and vertical coordinates. The equations governing small disturbances to this basic state are

$$
\begin{gathered}
\left(\left(\frac{\partial}{\partial t}+U \frac{\partial}{\partial x}\right) u^{\prime}+\frac{\mathrm{d} U}{\mathrm{~d} z} w^{\prime}\right)=-\frac{1}{\rho_{0}} \frac{\partial p^{\prime}}{\partial x}+\nu \nabla^{2} u^{\prime} \\
\left(\frac{\partial}{\partial t}+U \frac{\partial}{\partial x}\right) w^{\prime}=-\frac{1}{\rho_{0}} \frac{\partial p^{\prime}}{\partial z}+\nu \nabla^{2} w^{\prime}, \\
\frac{\partial u^{\prime}}{\partial x}+\frac{\partial w^{\prime}}{\partial z}=0,
\end{gathered}
$$

where $u^{\prime}, w^{\prime}$ and $p^{\prime}$ denote perturbation quantities of velocity and pressure from the mean values $U, 0$ and $p_{o}$, and $\nu$ is the kinematic viscosity. Equation (2.3) implies that we may define a perturbation stream function $\psi$ by

$$
u^{\prime}=-\frac{\partial \psi}{\partial z}, \quad w^{\prime}=\frac{\partial \psi}{\partial x}
$$

If $\zeta$ is the perturbation vorticity, (2.1)-(2.3) give the vorticity equation

$$
\left(\frac{\partial}{\partial t}+U \frac{\partial}{\partial x}\right) \zeta-w^{\prime} \frac{\mathrm{d}^{2} U}{\mathrm{~d} z^{2}}=\nu \nabla^{2} \zeta, \quad \zeta=\nabla^{2} \psi
$$

We look for disturbances in the form of normal modes, namely

$$
\psi=\hat{\psi} r(z) \mathrm{e}^{\mathrm{i} k(x-c t)}
$$

and substituting into (2.5) gives the Orr-Sommerfeld equation for $\hat{\psi}$ :

$$
(U-c)\left(\frac{\mathrm{d}^{2} \hat{\psi}}{\mathrm{d} z^{2}}-k^{2} \hat{\psi}\right)-\frac{\mathrm{d}^{2} U}{\mathrm{~d} z^{2}} \hat{\psi}=-\frac{\mathrm{i} \nu}{k}\left(\frac{\mathrm{d}^{4} \hat{\psi}}{\mathrm{d} z^{4}}-2 k^{2} \frac{\mathrm{d}^{2} \hat{\psi}}{\mathrm{d} z^{2}}+k^{4} \hat{\psi}\right) .
$$

For such flows the vertical displacement $\eta$ of a particle is

$$
\eta=\hat{\eta}(z) \mathrm{e}^{\mathrm{i} k(x-c t)}, \quad \text { where } \hat{\psi}=(U-c) \hat{\eta} .
$$




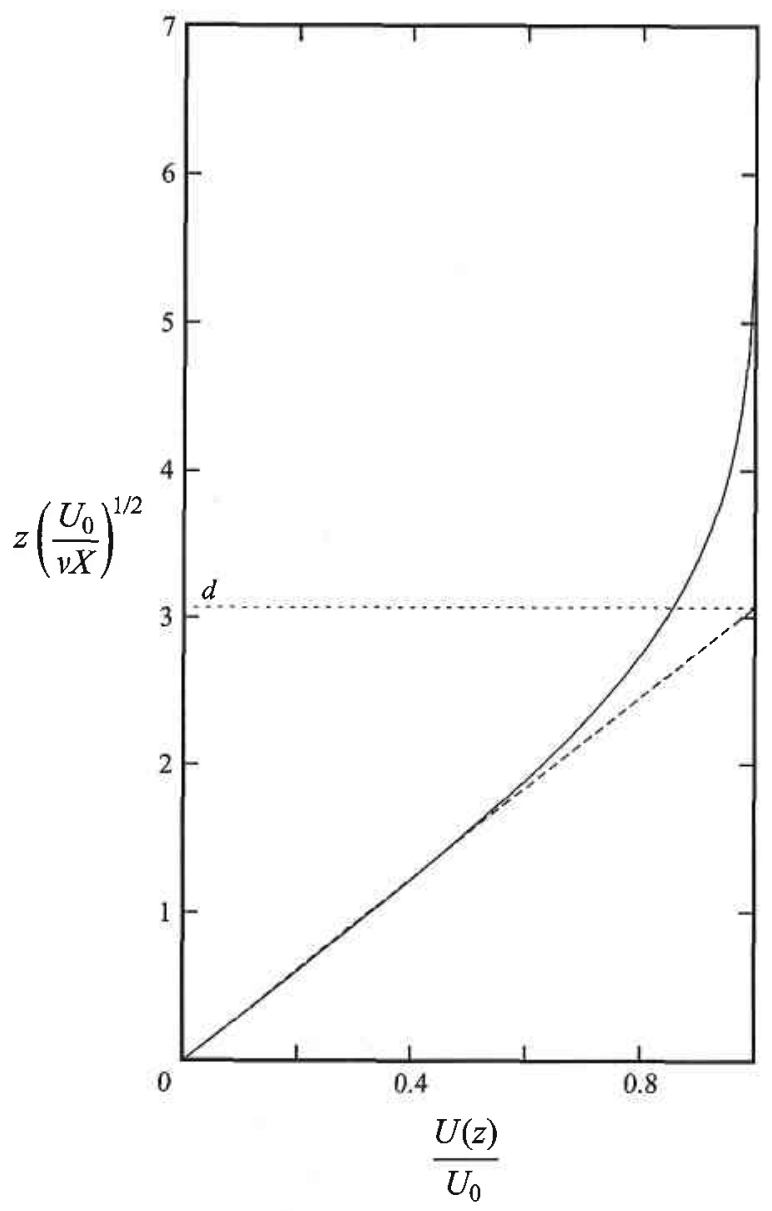

FIGURE 1 . The velocity profile of a Blasius boundary layer over a flat plate, together with the idealized profile (2.9) with $\delta=0$.

The velocity profile of a Blasius boundary layer, due to flow over a flat plate commencing at $X=0$ where $X$ is the coordinate along the plate, is shown in figure 1 . We may define a boundary-layer 'thickness' $d$ by the intersection of the two straight lines as shown. In practice $d=3.15\left(\nu X / U_{0}\right)^{1 / 2}$, and varies slowly with $X$, but it is assumed constant here (the 'parallel-flow approximation'). For present purposes it will be convenient to instead use analytically simpler profiles of the form

$$
\begin{aligned}
U(z) & =U_{0} z / d, & & 0<z<d-\delta, \\
& =U_{0}\left(1-\frac{(d+\delta-z)^{2}}{4 d \delta}\right), & & d-\delta<z<d+\delta, \\
& =U_{0}, & & z>d+\delta,
\end{aligned}
$$

which give a good approximation to the Blasius profile when $\delta / d=0.32$. These profiles are smooth except in the special case of $\delta=0$, where the central region disappears and there is a vorticity interface at $z=d$.

We next take non-dimensional variables $x^{*}, z^{*}$ and $t^{*}$ defined by and define

$$
x^{*}=x / d, \quad z^{*}=z / d, \quad t^{*}=t U_{0} / d,
$$

$$
\phi\left(z^{*}\right)=\hat{\psi} / U_{0} d, \quad \bar{u}\left(z^{*}\right)=U(z) / U_{0}, \quad C=c / U_{0}, \quad \alpha=k d, \quad \operatorname{Re}=U_{0} d / \nu,
$$


where $R e$ is the Reynolds number. Equation (2.7) then becomes

$$
(\bar{u}-C)\left(\frac{\mathrm{d}^{2} \phi}{\mathrm{d} z^{* 2}}-\alpha^{2} \phi\right)-\frac{\mathrm{d}^{2} \bar{u}}{\mathrm{~d} z^{* 2}} \phi=-\frac{\mathrm{i}}{\alpha \operatorname{Re}}\left(\frac{\mathrm{d}^{4} u}{\mathrm{~d} z^{* 4}}-2 \alpha^{2} \frac{\mathrm{d}^{2} \bar{u}}{\mathrm{~d} z^{* 2}}+\alpha^{4} \phi\right) .
$$

With the no-slip boundary condition at the wall, and the requirement that the disturbance be bounded as $z \rightarrow \infty$, the boundary conditions are

$$
\phi, \frac{\mathrm{d} \phi}{\mathrm{d} z^{*}}=0 \quad \text { at } \quad z^{*}=0 \quad \text { and } \quad \phi, \frac{\mathrm{d} \phi}{\mathrm{d} z^{*}} \rightarrow 0 \quad \text { as } \quad z^{*} \rightarrow \infty .
$$

The solution of this mathematical problem for the eigenvalues $C$ and the eigenfunctions $\phi$ goes back to Tollmien and Schlichting and is well-described in several texts (e.g. Stuart 1963; Schlichting 1968; Drazin \& Reid 1981), and the stability properties of the system are well known. Our purpose here is to describe the mechanics of this process. To do this, in the next two sections we examine the possible free modes that may exist in simple idealizations of the Blasius profile.

\section{The inviscid mode}

If (2.12) is simplified to the inviscid system $(R e=\infty)$ with the free-slip boundary condition, the idealized profile (2.7) with $\delta=0$ has one free mode with the form

with eigenvalues

$$
\begin{gathered}
\phi=\sinh \alpha z^{*} / \sinh \alpha, \quad 0 \leqslant z^{*} \leqslant 1, \\
=\mathrm{e}^{-\alpha\left(z^{*}-1\right)}, \quad z^{*} \geqslant 1, \\
C=1-\frac{\tanh \alpha}{\alpha(1+\tanh \alpha)} .
\end{gathered}
$$

All these wave speeds $C$ are therefore positive, ranging from 0 to 1 . This represents a wave on the vorticity interface at $z^{*}=1$, propagating against the flow and advected with it. Equation (3.2) is expected to give a good approximation to the dispersion relation for the corresponding wave on profiles (2.9) with $\delta$ up to about 0.3 , and for waves on the Blasius profile itself, provided that $\alpha$ and $C$ are small.

\section{Viscous modes in uniform shear}

We next consider modes in a velocity profile with uniform shear, namely

$$
U(z)=U_{z} z
$$

where $U_{z}=\mathrm{d} U / \mathrm{d} z$ is constant. The existence of such modes has been noted before (e.g. Grohne 1954; Corcos \& Sellars 1959), but not, apparently, their detailed properties that are required here. For this system the term $U_{z z}$ is absent from (2.7), and the resulting terms suggest that the appropriate length and time scales $L$ and $T$ are

$$
L=\left(\nu / k U_{z}\right)^{1 / 3}, \quad T=1 / U_{z},
$$

with speed scale $L / T=\left(\nu U_{z}^{2} / k\right)^{1 / 3}$. These modes are of interest in their own right, and hence we adopt this scaling for this section. Defining

and writing

(2.7) becomes

$$
z^{\prime}=z / L, \quad t^{\prime}=t / T, \quad c^{\prime}=c T / L, \quad \kappa=k L=\left(\nu k^{2} / U_{z}\right)^{1 / 3},
$$

$$
\Phi^{\prime}=\left(\frac{\partial^{2}}{\partial z^{\prime 2}}-\kappa^{2}\right) \hat{\psi}
$$

$$
\frac{\mathrm{d}^{2} \Phi^{\prime}}{\mathrm{d} Z^{\prime 2}}-Z^{\prime} \Phi^{\prime}=0,
$$




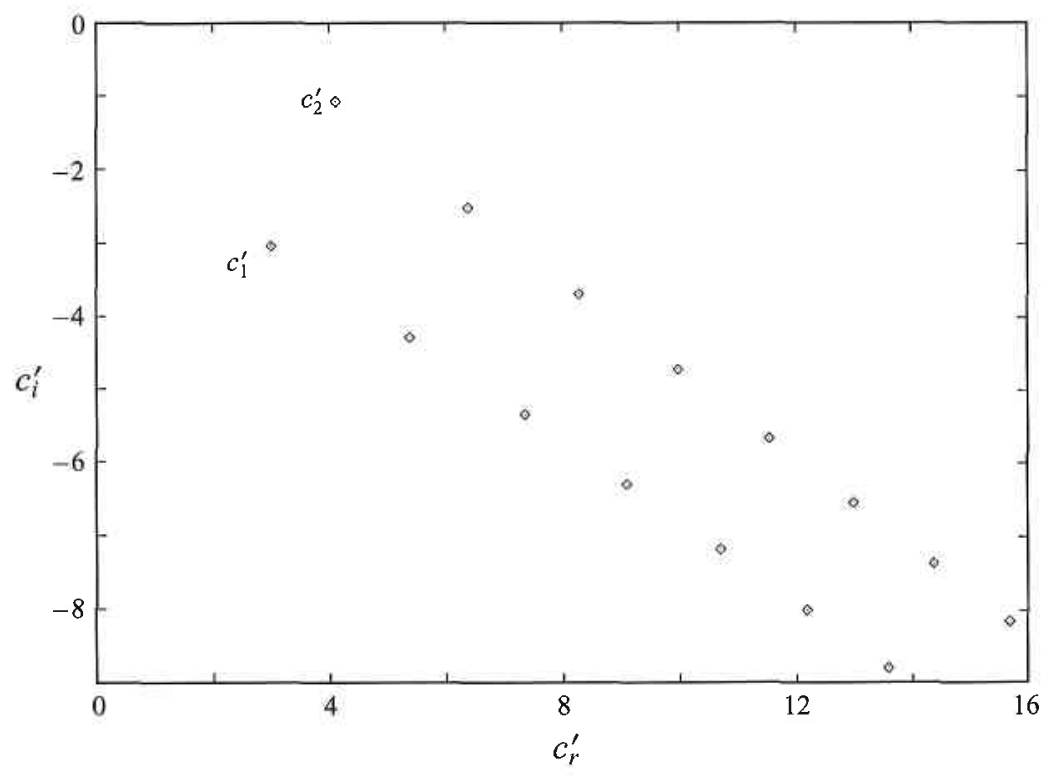

FIGURE 2. Complex wave speeds (eigenvalues) $c_{n}^{\prime}=c_{n r}^{\prime}+\mathrm{i} c_{n i}^{\prime}$ for decaying viscous modes in uniform shear, $n=1,2,3, \ldots$, for $\kappa=0.00677$.

where

$$
Z^{\prime}=-\left(\mathrm{i}\left(z^{\prime}-c^{\prime}\right)+\kappa^{2}\right)
$$

The solution to (4.5) that decays to zero when $z^{\prime} \rightarrow \infty$ is

$$
\Phi^{\prime}\left(z^{\prime}\right)=\operatorname{Ai}\left(Z^{\prime} \mathrm{e}^{\mathrm{i} 2 \pi / 3}\right)
$$

where $\mathrm{Ai}$ is the Airy function. From (4.4) $\hat{\psi}$ is given by

$$
\hat{\psi}=-\frac{1}{2 \kappa}\left(\int_{z^{\prime}}^{\infty} \Phi^{\prime}(\xi) \mathrm{e}^{\kappa\left(z^{\prime}-\xi\right)} \mathrm{d} \xi+\int_{0}^{z^{\prime}} \Phi^{\prime}(\xi) \mathrm{e}^{-\kappa\left(z^{\prime}-\xi\right)} \mathrm{d} \xi\right)+A \mathrm{e}^{-\kappa z^{\prime}}
$$

where $A$ is a constant. $A$ and $c^{\prime}$ are then determined by the no-slip conditions at $z^{\prime}=0$, namely $\hat{\psi}=0=\mathrm{d} \hat{\psi} / \mathrm{d} z^{\prime}$, which give $A=0$ and

$$
\int_{0}^{\infty} \Phi^{\prime}(\xi) \mathrm{e}^{-\kappa \xi} \mathrm{d} \xi=0
$$

This equation may be solved to obtain an infinite set of eigenvalues $c^{\prime}$ for each $\kappa$, which immediately give the corresponding eigenfunctions for the vorticity $\Phi^{\prime}$ and stream function $\hat{\psi}$ from (4.7) and (4.8). We therefore have an infinite discrete spectrum of free solutions, where the discretization is specified by the no-slip boundary conditions at $z^{\prime}=0$. These solutions do not appear to have been described before, although their presence is implicit in many treatments of boundary-layer disturbances (e.g. Drazin \& Reid 1981) and in triple-deck formulations (Smith 1979; Bogdanova \& Ryzhov 1983).

Writing $c^{\prime}=c_{r}^{\prime}+i c_{i}^{\prime}$, for all of these modes $c_{r}^{\prime}$ is positive and $c_{i}^{\prime}$ is negative, so that they decay with time. They are numbered in order of increasing values of $c_{r}^{\prime}$, and for the $n$th mode we write $c_{n}^{\prime}=c_{r n}^{\prime}+\mathrm{i} c_{i n}^{\prime}$. The values of $c_{n}^{\prime}$ for the first 15 modes are shown in figure 2 for $\kappa=0.00677$. These are very close to the values for $\kappa=0$ (and also for $\kappa=0.0353$ below). For most situations of interest in this paper $\kappa$ is very small $(<0.086)$, and the values in figure 2 are taken as representative for the present discus- 

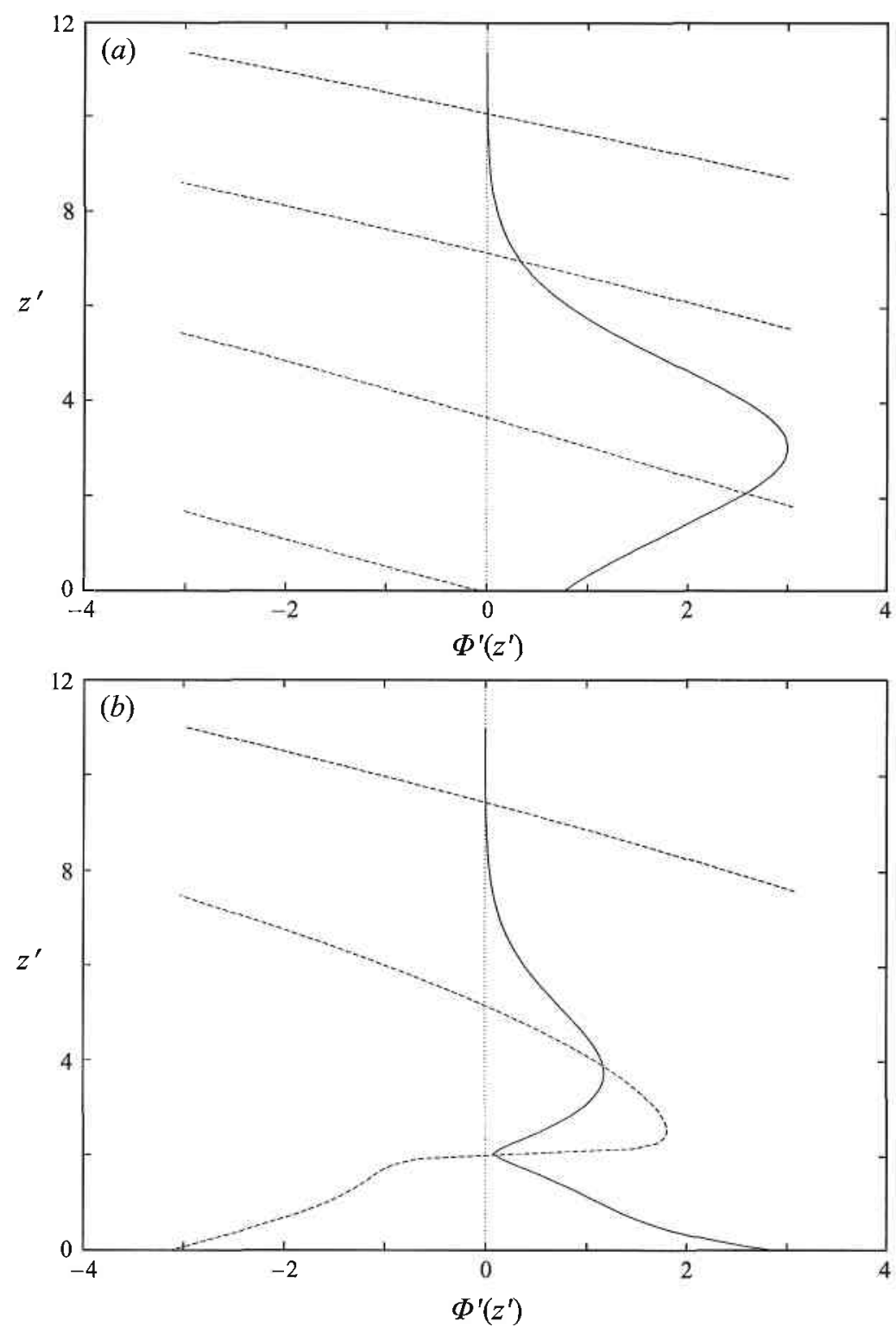

FIGURE 3. Amplitude (solid line) and phase (dashed) of the vorticity as a function of $z^{\prime}$ for the first mode of $(a)$ the lower family of figure 2 and $(b)$ the upper family, for $\kappa=0.0353$. The scale of the abscissa has a chosen maximum of three for the amplitude, and is in radians for the phase.

sion. The plotted values in figure 2 lie along two approximately straight lines. These lines identify two separate families of eigenfunctions, which derive from the symmetry properties of the Airy function about the real axis, and its oscillatory behaviour in the half-plane where the real part of the argument is negative. Figure 3 (plotted for $\kappa=$ 0.0353 ) shows the form of the vorticity for the first member of each family. For the members of the first or lower family in figure 2 , the lines of constant phase for vorticity have positive slope $\left(=\kappa / \arg \left(\operatorname{Ai}\left(Z^{\prime} \mathrm{e}^{2 \pi \mathrm{i} / 3}\right)\right)\right.$ in physical space $(x, z)$, and the first mode (depicted in figure $3 a$ ) has approximately uniform slope of $O(\kappa)$ from the wall upwards, over the distance shown. This slope decreases slowly with $z^{\prime}$ as $z^{\prime}$ becomes large, and 

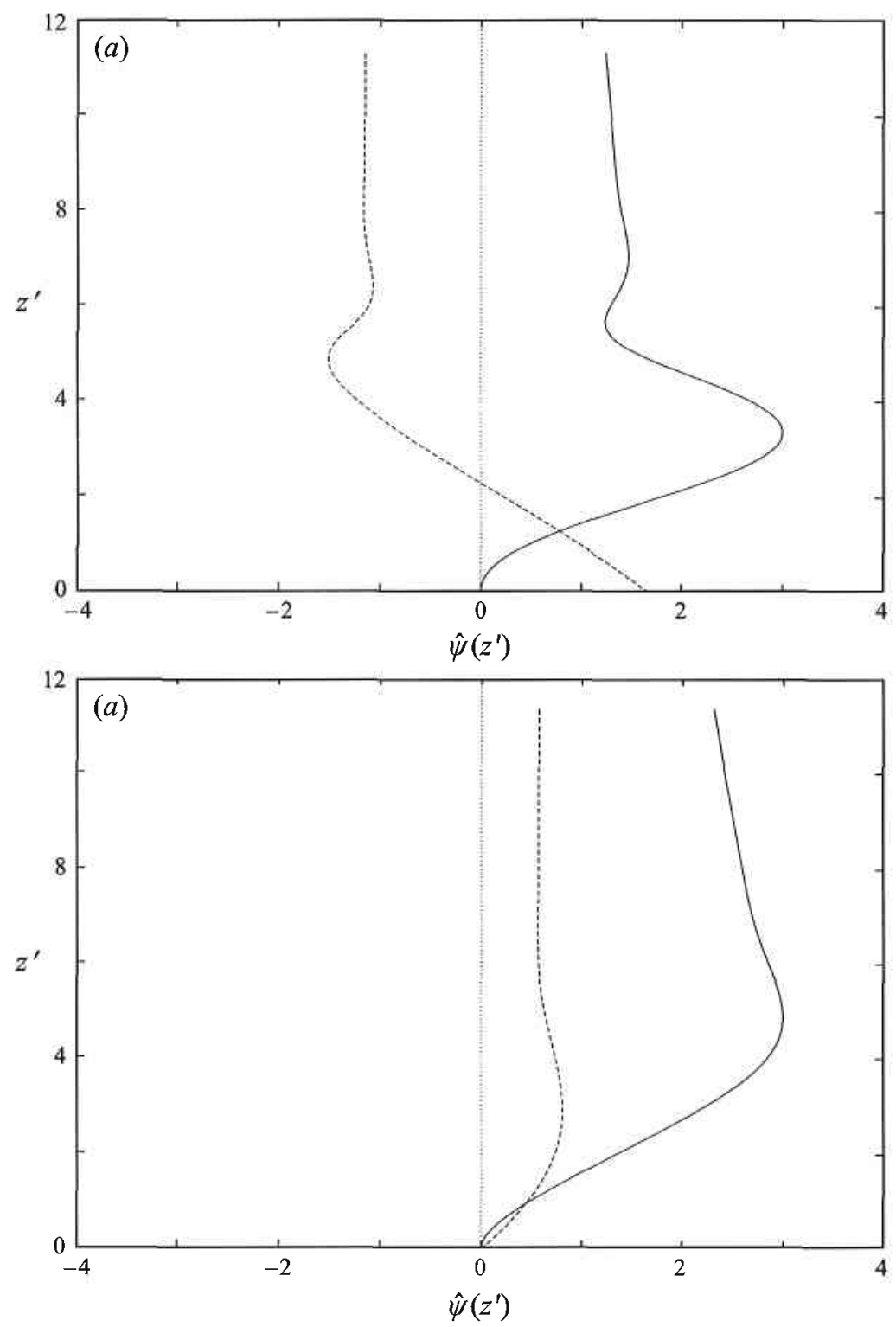

Figure 4. As figure 3 but showing the stream function.

does not appear to asymptote to a finite value. The net effect of uniform shear and viscosity on the vorticity of this periodic disturbance is therefore a decaying structure with positive slope of order $\kappa$, with diffusion preventing the structure from being advected to smaller slopes and scales by the shear, as would occur in an inviscid fluid (Phillips 1966; Hartman 1975).

For the second or upper family of modes in figure 2, for which the first member is depicted in figure $3(b)$, the slope of the phase lines for vorticity in physical space is negative at the wall. But at finite distances from it the vortex lines are advected and rotated by the shear, and this causes the slope of the phase lines to reverse sign at a distance where $c_{r} \approx U$. At still larger distances these lines approach a slope of order $\kappa$ as for the first family. A travelling stress pattern at the wall, with wavenumber $k$ and 

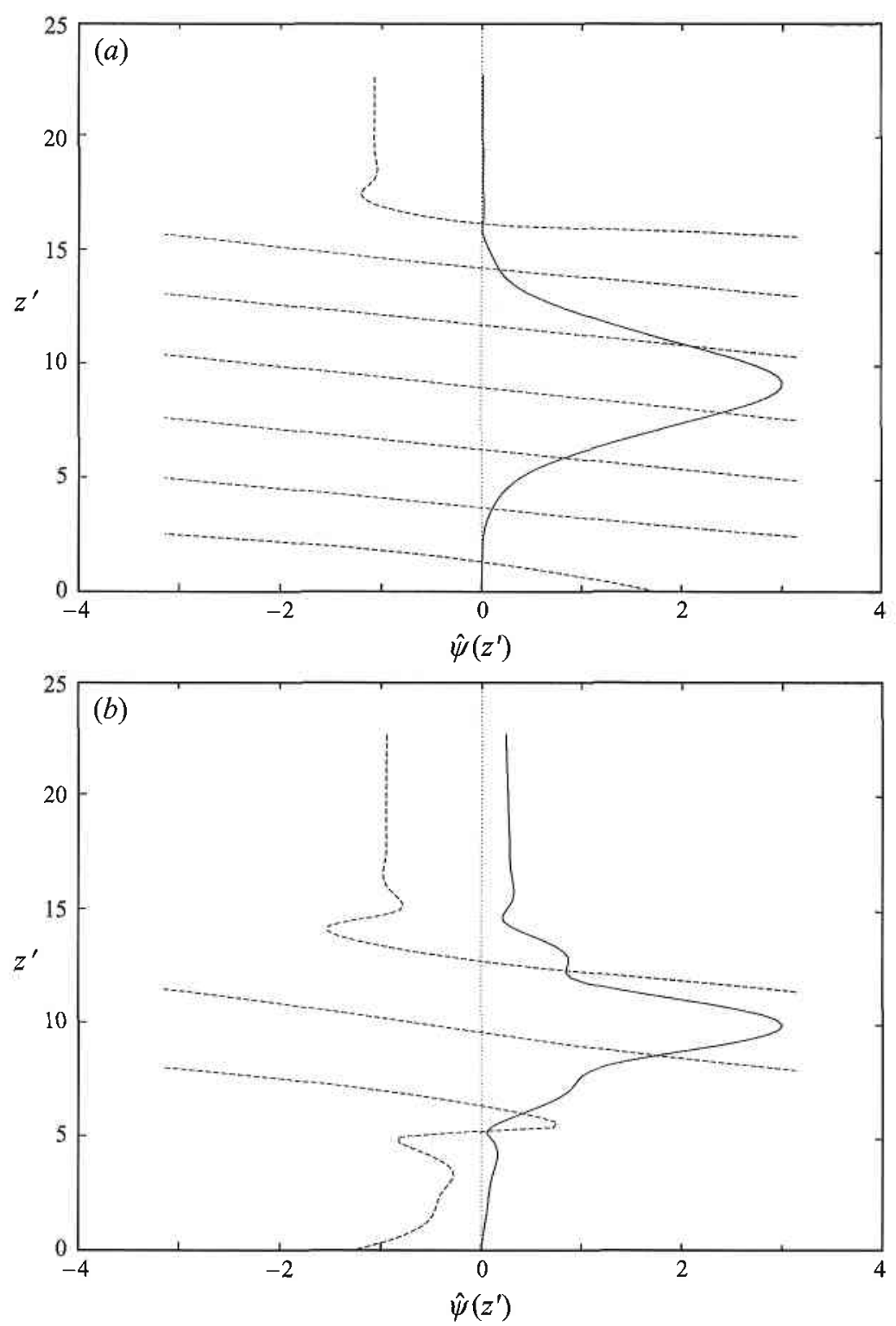

FIGURE 5. As figure 4, but for the fourth mode of $(a)$ the lower family, and $(b)$ the upper family; $\kappa=0.0353$.

positive speed $c$ in fluid at rest with no shear would produce phase lines with negative slope, diffusing away from the wall. The structure of the members of the second family of modes shows the effect of uniform shear on such motion.

From (4.8), the stream function $\hat{\psi}$ (and hence the velocity) extends beyond the range of $z^{\prime}$ where the vorticity $\Phi^{\prime}$ is significant, and has irrotational behaviour $\mathrm{e}^{-\kappa z^{\prime}}=\mathrm{e}^{-k z}$ far from the wall, with uniform phase. Amplitudes and phases for $\hat{\psi}$ for the first mode for each family are shown in figure 4 . Note that the first mode of the upper family, which has the smallest decay rate of all, has a simple amplitude structure and approximately uniform phase.

Examples of the structure of the higher modes are presented in figure 5, which shows 
the stream functions for the fourth mode for each family. The properties are similar to those for the first modes, although the main part of the mode is concentrated further from the wall, near where $c_{r}=U(z)$.

These modes are free disturbances that satisfy the no-slip boundary condition and have a non-zero stress pattern at the wall, travelling at the specified speed for given $k$. They may be generated by suitable initial conditions, and then decay exponentially with time without varying their structure. They are not 'wave modes', since they are governed by advection and diffusion of the disturbance vorticity $\left(2.7\right.$ with $\left.U_{z z}=0\right)$. However, they resemble damped wave modes in systems with restoring forces, and will be regarded as such in the discussion below. The reason why these discrete modes exist at all is not intuitively obvious, but one may interpret the spectrum in terms of the ratio of length scales. In addition to the intrinsic length scale $L$, a given wave speed $c$ gives an additional length scale $z_{c}$, the distance from the wall to the level where $U\left(z_{c}\right)=c_{r}$. The boundary conditions at the wall constrain the structure there, and coherent structures or 'resonances' are obtained when the distance $z_{c}$ is some suitable multiple of $L$. This results in the approximately evenly spaced eigenvalues for $c$.

For later reference we also need these modes in the notation of the nondimensionalization (2.10) and (2.11). Here equations (4.5)-(4.9) for the modes in viscous infinite shear become

$$
\frac{\mathrm{d}^{2} \Phi}{\mathrm{d} Z^{2}}-Z \Phi=0, \quad \Phi=\frac{\mathrm{d}^{2} \phi}{\mathrm{d} z^{* 2}}-\alpha^{2} \phi
$$

where

$$
\Phi\left(z^{*}\right)=A_{i}\left(Z \mathrm{e}^{\mathrm{i} 2 \pi / 3}\right), \quad Z=-(\alpha R e)^{1 / 3}\left(\mathrm{i}\left(z^{*}-C\right)+\alpha / R e\right) .
$$

The solution for $\phi$ in terms of $\Phi$ is

with

$$
\phi=-\frac{1}{2 \alpha}\left(\int_{z^{*}}^{\infty} \Phi \mathrm{e}^{\alpha\left(z^{*}-z^{\prime}\right)} \mathrm{d} z^{\prime}+\int_{0}^{z^{*}} \Phi \mathrm{e}^{-\alpha\left(z^{*}-z^{\prime}\right)} \mathrm{d} z^{\prime}\right),
$$

$$
\int_{0}^{\infty} \Phi(\xi) \mathrm{e}^{-\alpha \xi} \mathrm{d} \xi=0
$$

The eigenvalues may be expressed as

$$
C=C_{n}=C_{r n}+\mathrm{i} C_{i n}=\frac{c_{n}^{\prime}}{(\alpha R)^{1 / 3}}, \quad n=1,2,3, \ldots,
$$

where $c_{n}^{\prime}$ denotes the value of $c^{\prime}$ for the $n$th mode (see figure 2), and is approximately constant if $\kappa=\left(\alpha^{2} / R e\right)^{1 / 3}$ is small (the variation is a few percent for $\kappa$ in the range of interest, from zero to 0.086 ). For the second and most weakly damped mode, for $\kappa=0$,

$$
C_{r 2}+\mathrm{i} C_{i 2}=\frac{(4.13-1.06 \mathrm{i})}{(\alpha R e)^{1 / 3}} .
$$

The eigenvalues shown in figure 2 have a similar pattern to the eigenvalues for decaying modes for the Blasius profile, as described by Mack (1976) and Antar \& Benek (1978). For given $\alpha$ and $R e$, there is a finite number of the latter, with the speeds $c_{r} / U_{0}<1$. As $R e$ increases, so does the number of these modes; their values of $c / U_{0}$ also decrease (see Mack 1976, figure 2), and those for the lowest modes approach the values shown in figure 2 (with the appropriate value of $\kappa$ ). In other words, as the 
vertical scale of these modes of the Blasius profile decreases with increasing Re and they move closer to the wall, they become identifiable with the modes in uniform shear described above.

If a travelling velocity field of the form

$$
u=u_{0} \cos (k(x-c t)), \quad w=0
$$

is specified at $z=0$ in place of the no-slip condition, then the solution for $\Phi$ has the form

$$
\Phi=\frac{2 u_{0}}{U_{0}} \frac{\operatorname{Ai}\left(Z \mathrm{e}^{2 \pi i / 3}\right)}{\int_{0}^{\infty} \operatorname{Ai}\left(Z(\xi) \mathrm{e}^{2 \pi \mathrm{i} / 3}\right) \mathrm{e}^{-\alpha \xi} \mathrm{d} \xi} .
$$

The denominator is the same integral as in (4.9), with the difference that $c$ is now real and specified. Hence a maximum response is expected when $c$ is close to the complex zeros of (4.9). In fact, as seen below, the significance of the viscous modes of this section for the instability is that they represent coherent structures that may be excited in a resonant fashion by forcing at the boundary.

\section{Stability properties and growing modes of Blasius and Blasius-like profiles}

Growth rates and phase speeds for growing disturbances to the Blasius profile have been computed by several authors, and are summarized in Schlichting (1968). Figure 6 , taken from this book, shows the region of instability in the $(\alpha, R e)$-plane. Properties of the eigenvalues of other modes have been described by Mack (1976).

Here we investigate some properties of the simpler model profiles (2.9) that approximate the Blasius profile. This enables the flow profiles to be varied, and has the advantage that the structure of the eigenfunctions in the three parts of the profile may be expressed in terms of exponential and Airy functions, and hence related to the modes of $\S \S 3$ and 4 . Further, the procedure for finding the modes may be reduced to algebra by satisfying the boundary conditions at the junctions $z=d \pm \delta$. One approximation has been made, namely that in the term $(U-c)$ in (2.7) in the central region where $|z-d|<\delta, U$ has been approximated by a constant value $U_{0}(1-\gamma \delta / d)$, where $\gamma$ typically has a value of 0.25 . This approximation was tested by varying $\gamma$ near this value, and little change in the results was found.

The regions of instability on the $(R e, \alpha)$-plane for disturbances to the profile (2.9) are shown in figure 7 for various values of $\delta / d$. These stability properties are sensitive to the precise shape of the profile and are discussed in the next section, but for $\delta / d \approx 0.32$ where the profile resembles the Blasius profile, the region of instability in the $(R e, \alpha)$ plane resembles that shown in figure 6 . However, the upper branch of the curve for $\delta / d=0.32$ lies substantially above that for the Blasius profile. This difference may be attributed to the absence of the effect of non-zero $U_{z z}$ at the critical layer in the idealized profile. The inclusion of this effect would result in the damping of the 'inviscid' vorticity mode, and also cause changes to the interaction across the critical level that are proportional to $U_{z z}$. These effects can stabilize the flow at larger $\alpha$. As $\delta / d$ decreases to zero the region of instability shrinks and contracts toward larger Reynolds number. It appears to disappear altogether in the limit $\delta \rightarrow 0$, so that the flow is apparently stable for any $R e$ for sufficiently small $\delta$, and for finite $\delta$ if $R e$ is less than some upper bound. 

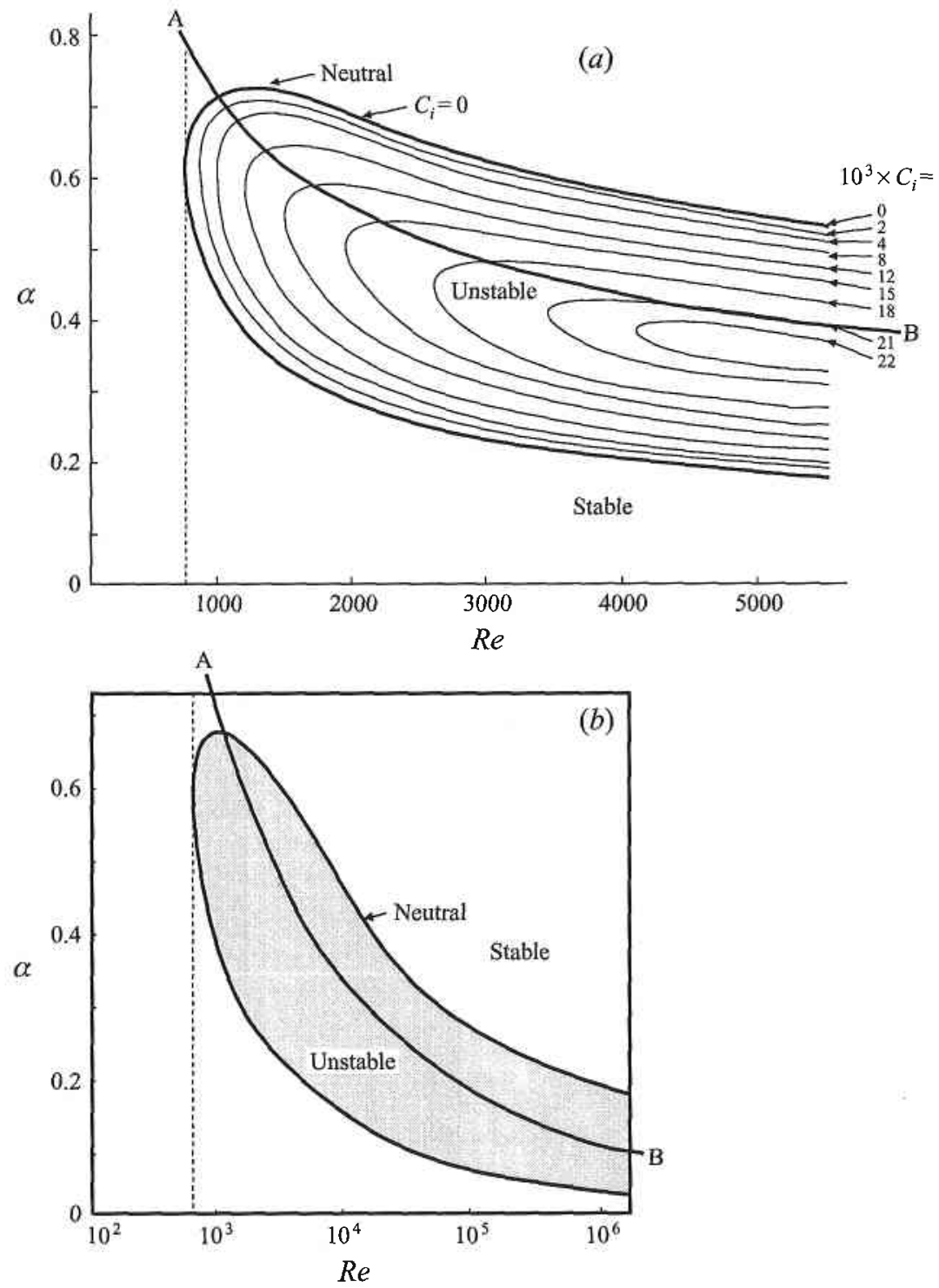

FIGURE 6. Regions of instability of a Blasius boundary layer on the $(R e, \alpha)$-plane: $(a)$ linear scale for $R e,(b)$ logarithmic scale. The line AB denotes (5.1), and the vertical dashed line denotes the critical value of Re. Modified from Schlichting (1968). Note that the Reynolds number and wavenumber in these diagrams in Schlichting (1968) are defined in terms of the displacement thickness $\delta_{1}$ rather than $d$, where $\delta_{1}=0.55 d$. (The neutral curves in the two figures are in fact incompatible near $\alpha=0.4$, indicating the uncertainty at the time of computation.)

We may expect that the modes for the idealized situations in $\S \S 3$ and 4 may have a presence in some modified form in modes for the Blasius and near-Blasius profiles, and we term them partial modes here. If one equates the speed (3.2) for the inviscid mode of $\S 3$ with the speed $C_{r 2}$ from (4.15) for the most weakly damped viscous mode of $\S 4$, one obtains the following relation between $\alpha$ and $R e$ :

$$
R e=\frac{69.93}{\alpha\left(1-(1 / 2 \alpha)\left(1-\mathrm{e}^{-2 \alpha}\right)\right)^{3}} .
$$




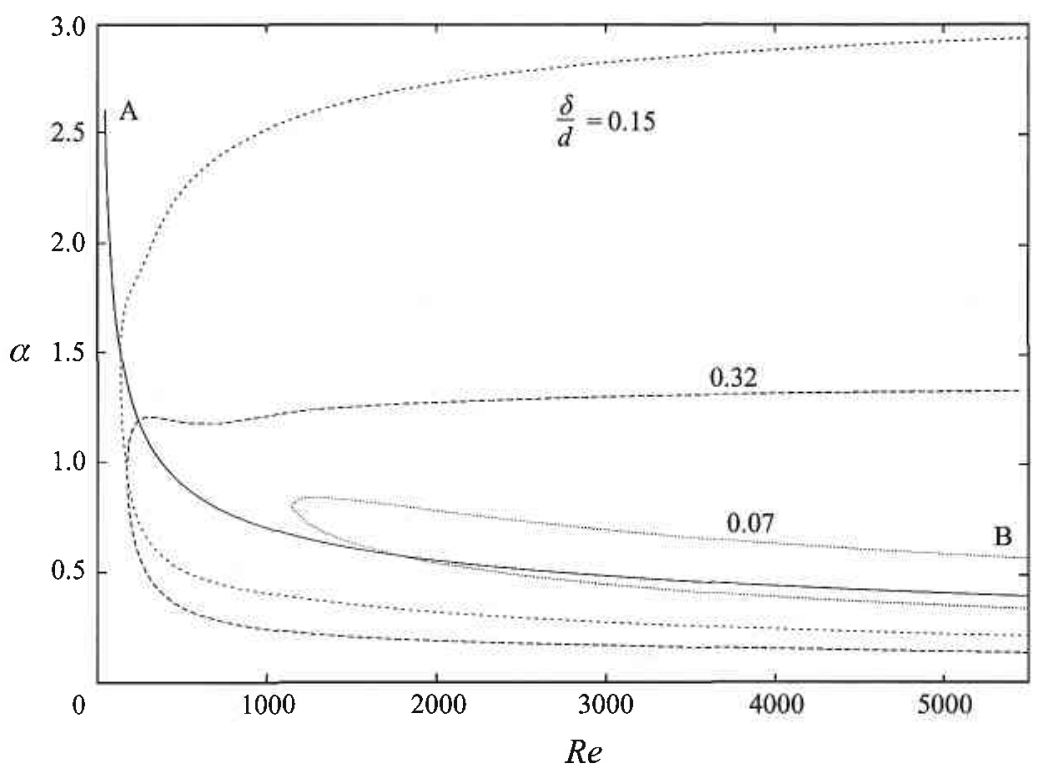

FIGURE 7. Boundaries of instability on the $(R e, \alpha)$-plane for velocity profiles (2.9) for values of $\delta / d$ as shown.

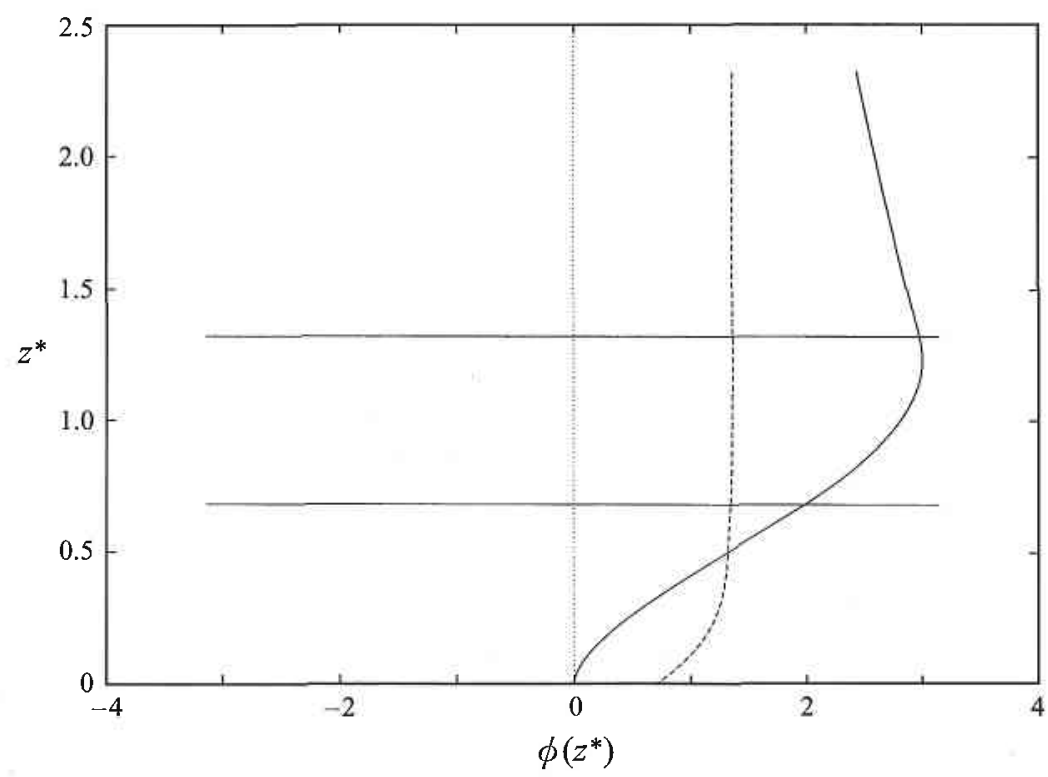

FIGURE 8. Form of the stream function of a typical eigenfunction for a growing mode for the model profile (2.9) with $\delta / d=0.32$ in the unstable region; $R e=3636, \alpha=0.2$. The solid line denotes amplitude and the dashed line phase, with the scale of the abscissa as in figure 3 . The horizontal lines denote $z=d \pm \delta$.

This relation is shown plotted in figures 6 and 7 as the curve $\mathrm{AB}$. The fact that (5.1) passes through approximately the centre of the unstable region for the Blasius boundary layer, close to the line of maximum growth rate, strongly suggests that the instability obtained by solving the full eigenvalue problem is caused by mutual 
interaction between these two free partial modes, which may be expected to be manifested in some form in the growing disturbances. In the next section we discuss the mechanics of this process.

Figure 8 shows the structure of the stream function for a representative growing mode for the profile (2.9) with $\delta / d=0.32$. This mode was computed for a number of other values of $R e$ and $\alpha$ in the unstable region, but these all had the same simple structure for amplitude and phase.

\section{The mechanism of instability}

For many unstable inviscid shear flows, for both homogeneous and density-stratified fluids, the essential kinematic mechanism of instability may be described in terms of the interaction of two 'partial modes' of the system (Sakai 1989; Baines \& Mitsudera 1994; Baines 1995). For these unstable systems the flow profile may be separated into two stable wave guides, each of which supports neutral waves when in isolation, and where waves in one waveguide may propagate in the opposite direction to waves in the other. From an energetic viewpoint these neutral waves have positive and negative energy (Craik 1985); and they are the 'partial modes' in the complete system. We may extend these notions here to explain Tollmien-Schlichting waves in the same way.

For the Blasius and near-Blasius boundary-layer profiles, the inviscid mode of $\S 3$ and the viscous decaying modes of $\$ 4$ may be regarded as 'partial modes' in a generalized sense; under suitable conditions each may approximate a complete mode of the system, but the viscous modes are concentrated close to the wall (if $R e \gg 1$ ), and the inviscid mode (most significantly) fails to satisfy the no-slip boundary condition. Since the results of the previous section indicate that instability results from their interaction, we investigate how this may occur.

We begin by imagining that an inviscid partial mode is present, propagating in the fluid with a Blasius or near-Blasius profile, with a stream function $\psi_{i}$ given approximately by (3.1) for $z^{*}$ not near 1 , and a dispersion relation given approximately by (3.2). This motion will have a non-zero velocity at the wall. If the no-slip condition there is to be satisfied by the nett resulting motion, the horizontal velocity at $z=0$ must be annuled by additional viscously forced motion, $\psi_{v}$. We may assume that this motion has the form (4.16), and the response that this forcing produces in a uniformly sheared viscous fluid has vorticity $\Phi$ given by (4.17), and stream function $\psi_{v}=\phi \mathrm{e}^{\mathrm{i} k x}$ where $\phi$ is given by

$$
\phi\left(z^{*}\right)=-\frac{A_{0}}{\alpha}\left(\sinh \alpha z^{*} \int_{z^{*}}^{\infty} \mathrm{Ai} \mathrm{e}^{-\alpha \xi} \mathrm{d} \xi+\mathrm{e}^{-\alpha z^{*}} \int_{0}^{z^{*}} \mathrm{Ai} \sinh \alpha \xi \mathrm{d} \xi\right),
$$

where

$$
\mathrm{Ai}=\mathrm{Ai}\left(Z(\xi) \mathrm{e}^{2 \pi \mathrm{i} / 3}\right), \quad Z(\xi)=-(\alpha \cdot R)^{1 / 3}\left(\mathrm{i}(\xi-C)+\frac{\alpha}{R e}\right)
$$

and

$$
A_{0}=u_{0} d / \int_{0}^{\infty} \mathrm{Ai}^{-\alpha \xi} \mathrm{d} \xi
$$

(These equations may alternatively be expressed in terms of $\kappa$ and $c^{\prime}$.) Here $C=c / U_{0}$ is real, and is determined by the speed of the (notional) inviscid mode. Equation (6.1) is very similar to (4.12), the only difference being that some exponentials are replaced by $\sinh$ functions. A typical example of $\phi\left(z^{*}\right)$ from (6.1) for $C$ near $C_{r 2}$ is shown in 


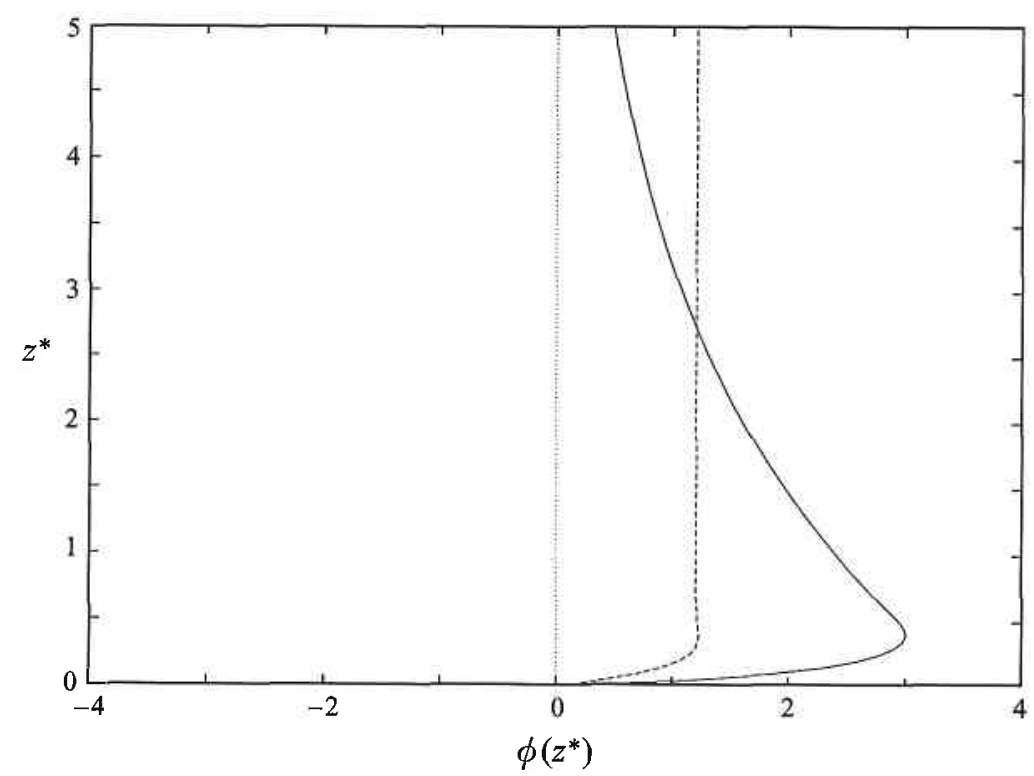

FIGURE 9. Profile of the stream function for the motion forced by a travelling periodic velocity at $z=0$ as given by equation (6.1), travelling at given speed $C=0.35$ with $\alpha=0.4, R e=3636$. The solid line denotes amplitude and the dashed line phase, as in figure 3.

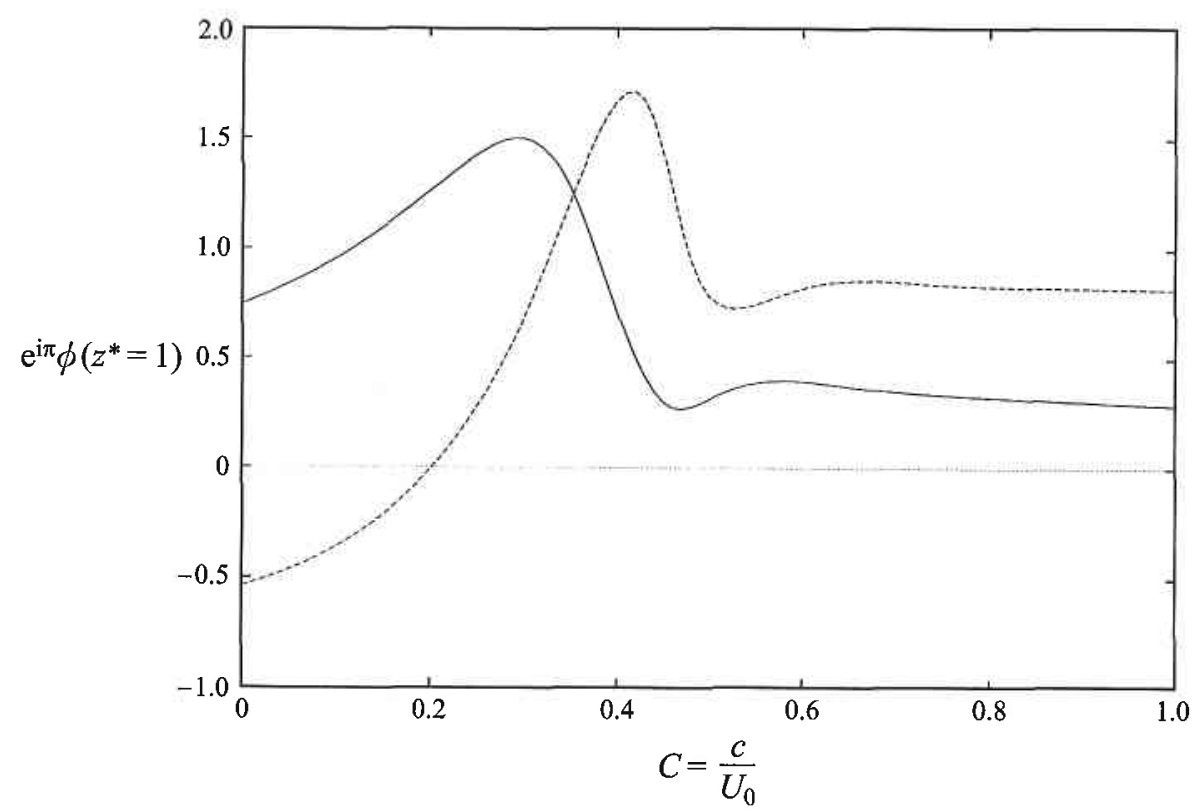

Figure 10. Amplitude (solid line) and phase (dashed line) at $z^{*}=1$ of the stream function of the forced viscous response to periodic velocity at $z=0$, travelling at speed $C . \alpha=0.4, \operatorname{Re}=3636$. The amplitude scale is again arbitrary, and the scale of the phase is in radians. (Note that the ordinate is $-\phi(1)$, not $\phi(1))$.

figure 9. The magnitude of the forced response (6.1) is sensitive to the value of $C$, and we may expect near-resonant behaviour when $C$ is close to $C_{r n}$ for $n=1,2,3 \ldots$ An example of the variation of $\phi(1)$ with $C$ is shown in figure 10 . There is a single major peak between $C_{r 1}$ and $C_{r 2}$, which implies that these two potential resonances overlap, 


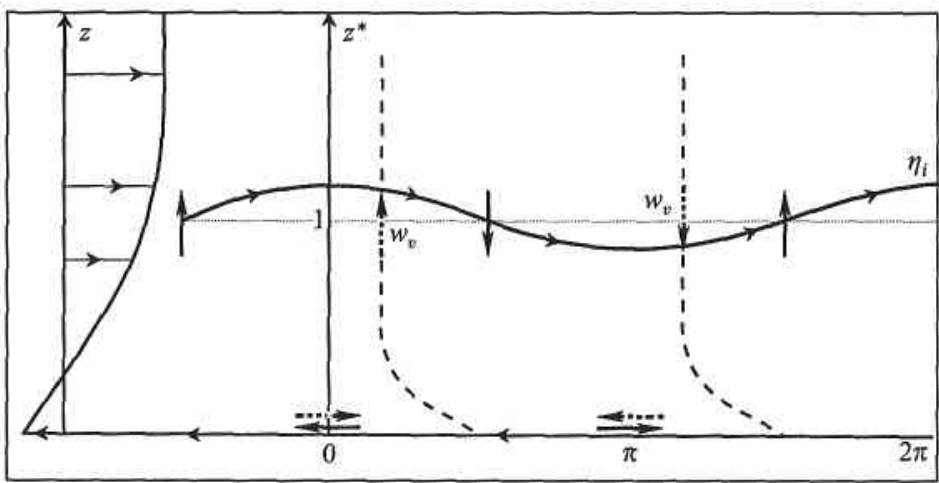

FIGURE 11. Schematic diagram of the interaction process that causes growth in a Tollmien-Schlichting wave. The flow is drawn in the frame of reference moving with the wave, so that the pattern is stationary but growing. The mean velocity profile in this frame is shown on the left. $\eta_{i}$ denotes the vertical displacement of the vortical region of the inviscid partial mode propagating on the vorticity gradient. In this partial mode the fluid at the boundary $z=0$ has the velocity denoted by the solid arrows, and the no-slip condition forces a viscous response represented by the dashed arrows. The dashed curves show the phase of this viscous response for the maxima in vertical velocity $w_{v}$. Since this is close to the maxima in $\eta_{i}$, it increases the amplitude of the vortical partial mode by advection.

and a second peak near $C_{r 3}$. Equation (6.1) cannot be expressed solely in terms of the free modes of $\S 4$, but will have a significant projection on them, particularly on the $n$th mode when $C$ is close to $C_{r n}$. Close to the wall, where the mean shear has had little effect on the disturbance, the slope of the vortex lines will be negative, but this slope will become positive further from the wall as these lines are rotated by the shear. Hence we expect the modes of the second family (figures $3 b, 4 b$ ) to be more prominent in this motion than the modes of the first family.

If we take axes moving with the initial inviscid wave at speed $c$, we may write $\eta_{i}=\eta_{I} \mathrm{e}^{i k x}$ for the vertical displacement of the vortical region ( = vorticity interface for (3.1)), where $\eta_{I}>0$. This is related to the velocity field of the inviscid mode by (2.8) and (approximately) (3.1). To satisfy the no-slip condition at the wall, this forces

$$
\psi_{v}=\phi\left(z^{*}\right) \mathrm{e}^{\mathrm{i} k x}
$$

where $\phi\left(z^{*}\right)$ is given by (6.1)-(6.3) with $u_{0}>0$. For $z^{*} \gtrsim 1$

$$
\phi\left(z^{*}\right) \sim-|B| \mathrm{e}^{-\alpha z^{*}} \mathrm{e}^{\mathrm{i} \beta},
$$

where $B$ is a constant. Figure 10 shows $\phi\left(z^{*}=1\right.$ ) as a function of $C$ for $\alpha=0.4$ (and $R e=3636$ ). From (3.2) the inviscid partial mode has speed $C=0.33$, and for this value $\beta \approx 1.3$ radians. The associated velocity field is then

$$
w_{v}=\mathrm{i} k \phi\left(z^{*}\right) \mathrm{e}^{\mathrm{i} k x},
$$

and if we superimpose this velocity field on the initial wave, it is approximately in phase with the displacement $\eta_{i}$ of the vortical region. Since these two flow patterns are stationary in this frame of reference, this velocity $w_{v}$ will tend to increase $\eta_{I}$ by advection. This will increase the overall amplitude of the inviscid mode $\psi_{i}$ and hence in turn $\psi_{v}$, provided the effect is large enough to overcome the internal viscous dissipation. Figure 9 bears a general resemblance to the unstable mode in figure 8 , and this is not altered if an inviscid mode is added to the motion in figure 9 , since the latter has no phase variation with $z^{*}$.

This interaction process is shown in schematic form in figure 11 , and parallels the mechanism described for inviscid fluids in Baines \& Mitsudera (1994). It should be 
involved in the interaction process. Its presence simply ensures that the phase speeds of the viscous and vortical modes have opposite signs to each other.

In summary, the evidence for the veracity of this physical picture is as follows.

(i) If one equates the speed of the inviscid partial mode with the most weakly damped viscous partial mode in uniform shear, one obtains (5.1), curve AB in figure 6 . Since this applies over the whole range of $\alpha$ and $R e$, it implies that the instability process derives from the interaction between these modes in some way.

(ii) The phase of the forced viscous response to an inviscid partial mode is as required to give mutual forcing, and its amplitude becomes large through resonance, when the wave speed approximately coincides with the most weakly dampled viscous partial mode. The mutual forcing must also be strong enough to overcome the inherent damping due to viscosity.

(iii) The structure of the eigenfunctions for growing modes in the Blasius-like profiles (figure 8), including the variation of phase with $z$, approximately resemble a superposition of an inviscid partial mode plus the viscous forced response.

(iv) The concept of instability in shear flows through the mutual interaction of 'partial modes', and in particular by the advection of one mode by the velocity field of another, has been demonstrated elsewhere for inviscid flows (Craik 1985; Sakai 1989; Baines \& Mitsudera 1994; Baines 1995).

Most of this work was carried out at CSIRO Aspendale, where Humio Mitsudera was funded by grants from the Australian Research Council and Shell Australia. The authors are also grateful to Bill Saric for drawing our attention to Prandtl's work, and to a referee for pointing out the significance of critical layers and the 'upper branch' structure, and earlier references to the viscous modes of $\S 4$.

\section{REFERENCES}

ANTAR, B. N. \& BENEK, J. A. 1978 Temporal eigenvalue spectrum of the Orr-Sommerfeld equation for the Blasius boundary-layer. Phys. Fluids 21, 183-189.

BaINES, P. G. 1995 Topographic Effects in Stratified Flows. Cambridge University Press.

BAines, P. G. \& Mitsudera, H. 1992 On the mechanism of shear flow instability in stratified fluids and boundary-layers. Proc. 11th Austral. Fluid Mech. Conf., Univ. of Tasmania, Hobart, pp. $75-78$.

Baines, P. G. \& Mitsudera, H. 1994 On the mechanism of shear flow instabilities. J. Fluid Mech. $276,327-342$.

Betchov, R. \& Criminale, W. O. 1967 Stability of Parallel Flows. Academic.

Bogdanova, E. V. \& Ryzhov, O.S. 1983 Free and induced oscillations in Poiseuille flow. $Q$. $J$. Mech. Appl. Maths 36, 27-87.

Corcos, G. \& Sellars, J. R. 1959 On the stability of fully developed flow in a pipe. J. Fluid Mech. $5,97-112$.

Craik, A. D. D. 1985 Wave Interactions and Fluid Flows. Cambridge University Press.

Drazin, P. G. \& REID, W. H. 1981 Hydrodynamic Stability. Cambridge University Press.

GroHNE, D. 1954 Über das specktrum bei eigen schwingungen ebener laminarströmungen. $Z$. Angew Math. Mech. 34, 344-357.

HARTMAN, R. J. 1975 Wave propagation in a stratified shear flow. J. Fluid Mech. 71, 89-104.

Lighthill, M. J. 1963 Introduction. Boundary layer theory. In Laminar Boundary Layers (ed. L. Rosenhead), pp. 46-113. Oxford University Press.

LINDZEN, R. S. 1988 Instability of plane parallel shear flow (towards a mechanistic picture of how it works). Pageoph. 126, 103-121.

Lindzen, R. S. \& RAMBALdi, S. 1986 A study of viscous Poiseuille flow. J. Fluid Mech. 165, 355-372. 
MACK, L. M. 1976 A numerical study of the temporal eigenvalue spectrum of the Blasius boundary layer. J. Fluid Mech. 73, 497-520.

Phillips, O. M. 1966 The Dynamics of the Upper Ocean. Cambridge University Press.

RAYLEIGH, LORD 1896 The Theory of Sound, vol. II. Reproduced by Dover (1945).

SAKAI, S. 1989 Rossby-Kelvin instability: a new type of ageostrophic instability caused by a resonance between Rossby waves and gravity waves. $J$. Fluid Mech. 202, 149-176.

Schlichting, H. 1968 Boundary-Layer Theory. McGraw-Hill.

Sмiтн, F. T. 1979 On the non-parallel flow instability of the Blasius boundary-layer. Proc. R. Soc. Lond A 366, 91-109.

StUART, J. T. 1963 Hydrodynamic stability. In Laminar Boundary Layers (ed. L. Rosenhead), pp. 492-579. Oxford University Press.

TAKeHIRO, S. \& HAYASHI, Y. 1992 Over-reflection and shear instability in a shallow-water model. J. Fluid Mech. 236, 259-279. 